\title{
Ueber eine quantitative Eiweissspaltung durch Salzsäure.
}

\author{
(II. Mittheilung.) \\ Von
}

Prof. Dr. Rudolf Coln.

(Aus dem Laboratorium für Pharmakologie u. med. Chemie zu Königsberg i. P.).

(Der'Redaction zugegangen am 16. November 1898.)

Im XXII. Bande der Zeitschr. f. physiol. Chemie, S. 153 bis 175 , habe ich über Versuche berichtet, die Spaltungsprodukte des Eiweisses (Casein) in grösserem Umfange zu isoliren, als es bei den bisherigen Spaltungsversuchen geglückt war. Die Bedeutung derartiger Versuche liegt hauptsächlich darin, dass man erst dann zur Erkenntniss der Constitution des Eiweisses wird gelangen können, wenn man genau weiss, aus welchen Bausteinen und in welchem gegenseitigen quantitativen Verhältniss sich dasselbe zusammensetzt. Erst dann wird sich auch ein Weg finden lassen, auf dem man mit einiger Aussicht auf Erfolg zum künstlichen Aufbau des Eiweisses wird vorschreiten können. Die Methode sowohl, deren ich mich zur Spaltung bediente, als auch die Darstellung der einzelnen Spaltungsprodukte wichen in einigen Punkten von den bisher üblichen $a b$. Ich wandte statt verdünnter Salzsäure reine concentrirte rauchende an, kochte nicht $3 \times 24$ Stunden, sondern nur 5 Stunden und liess das seit den Versuchen von Hlasiwetz und Habermann behufs Vermeidung dunkelgefärbter, durch Oxydation entstehender Zersetzungsprodukte zugesetzte Zinnchlorür ganz fort. Ferner verzichtete ich darauf; durch Eindampfen der salzsauren Lösung der Eiweissspaltungsprodukte zuerst die Glutaminsäure als salzsaures Salz zu isoliren, sondern entfernte von vornherein die gesammte Salzsäure durch Kupferoxydul, was bis auf Spuren gelang, erhielt dann durch fractionirtes Eindampfen und Krystallisirenlassen eine Reihe von Fractionen freier Amidosäuren (Tyrosin und Leucin), und nachdem ich soweit 
eingedampft hatte, dass die dickflüssige Mutterlauge durch Filtriren von den ausgeschiedenen Krystallen nicht mehr gut zu trennen war, zerlegte ich das Gemenge durch Zusatz des ca. sechsfachen Volumens Alkohol in eine in diesem unlösliche und eine lösliche Portion, die ich dann gesondert auf die in ihnen enthaltenen einzelnen Spaltungsprodukte zu untersuchen gedachte. (Näheres siehe in meiner I. Mittheilung.) Unter den flüchtigen Spaltungsprodukten wies ich geringe Mengen $\mathrm{CO}_{2}$ und eines die Jodoformreaction gebenden Körpers (vielleicht Aceton) nach. Ausserdem fand sich unter den Zersetzungsprodukten ein schön krystallisirender Körper, dessen Analyse zu der vorläufigen Formel $\mathrm{C}_{5} \mathrm{H}_{7} \mathrm{NO}$ führte und den ich als ein Pyridinderivat glaubte ansprechen $z u$ dürfen. Weitere Untersuchungen über denselben, die zu einem interessanten Ergebnisse zu führen scheinen, sind im Gange und sollen später publicirt werden.

Im Ganzen erhielt ich über 91\% des angewandten Caseins an Zersetzungsprodukten und ich will gleich hier erwähnen, dass ich in späteren Versuchen durch besseres Auskochen und Auswaschen der grossen Niederschläge von Kupferchlorür und Schwefelkupfer es bis auf 97,8\% bringen konnte, eine Ausbeute, die man wohl in der That als quantitativ ansehen kann und die weit über die hinausgeht, welche frühere Untersucher erreicht haben. Im Einzelnen muss ich noch bemerken, dass ich sowohl Tyrosin als auch Leucin in sehr viel grösseren Mengen erhielt, ersteres in über 3,5\% (gegen $2 \%$ der früheren Forscher) und Leucin zunächst schon in über 31\% des verarbeiteten Caseins, wobei die Mengen desselben, die in den beiden noch nicht untersuchten Hauptantheilen, die ich durch Zusatz der sechsfachen Menge Alkohols getrennt hatte (siehe oben), enthalten waren, und über deren Verarbeitung ich im Folgenden das Nähere mittheilen werde, nicht mit eingerechnet sind.

Die vorliegende Mittheilung soll sich nun hauptsächlich mit der Zerlegung der eben erwähnten beiden Fractionen beschäftigen. Um es der Deutlichkeit wegen nochmals zu wiederholen, so hatte ich nach Abscheidung des Tyrosins und des durch einfaches Auskrystallisiren erhaltenen Leucins den dickflüssigen 
Rückstand in etwas Wasser klar gelöst und mit der ca. sechsfachen Alkoholmenge gefällt. Es fiel dabei ein in Alkohol unlösliches, dickes Oel aus, die sogenannte ölige Säure, von der ich vermuthete, dass sie ein Gemenge darstellte (die in der I. Mittheilung beschriebenen Versuche, mit Hülfe der Ueberführung in ein Cu-Salz zum Ziele zu gelangen, hatten noch kein bestimmtes Resultat ergeben; mit Sicherheit waren nur geringe Mengen Asparaginsäure darin nachgewiesen), und es blieb ein erheblicher Antheil in dem Alkohol in Lösung, mit dem eventuell ein durch Umfällen der öligen Säure gewonnener Antheil vereinigt wurde. Der Alkohol wurde darauf abdestillirt, der Rückstand zur Gewinnung des Pyridinderivats nochmals in Wasser gelöst, von dem darin unlöslichen Pyridinderivat abfiltrirt und die wässerige Lösung zur Trockene verdampft. Die Verarbeitung dieses so erhaltenen letzten Rückstandes geschah nun in folgender Weise :

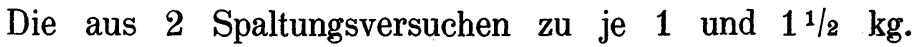
Casein erhaltenen, also $21 / 2 \mathrm{~kg}$. Casein entsprechenden letzten Rückstände, die einen dicken, halbfesten Syrup bildeten, wurden in kaltem Wasser gelöst, filtrirt und das 2 l. betragende Filtrat zur Gewinnung der basischen Bestandtheile fractionirt mit Phosphorwolframsäure gefällt, bis nichts mehr ausfiel. Es waren dazu verhältnissmässig grosse Mengen Phosphorwolframsäure erforderlich, da das Ammoniak vorher nicht entfernt war. Die I. Fällung erhielt ich durch 500 gr. Phosphorwolframsäure in 5 l. Wasser gelöst, der Niederschlag wurde auf einer Nutsche abgesogen und mit 5\% iger Schwefelsäure mehrmals ausgewaschen. Das Filtrat (ohne Waschwasser) wurde mit $500 \mathrm{gr}$. in 4 1. Wasser gefällt, der Niederschlag II ebenfalls abfiltrirt und ausgewaschen, das Filtrat schliesslich mit $2 \mathrm{~kg}$. Phosphorwolframsäure in $15 \mathrm{l}$. Wasser vollständig ausgefällt. Niederschlag I war braun und etwas schmierig, II fast farblos und feinpulverig, III, der reichlichste, farblos und ziemlich grobpulverig, sehr viel leichter auszuwaschen. Die 3 Niederschläge wurden gesondert, nachdem sie zunächst mit kochendem Barytwasser zersetzt, aus dem Filtrat der Baryt möglichst genau mit $\mathrm{H}_{2} \mathrm{SO}_{4}$ ausgefällt, die Filtrate mit $\mathrm{HCl}$ angesäuert und eingedampft waren, nach 
den üblichen Methoden auf Basen verarbeitet. Diese Untersuchung, deren Einzelheiten ich übergehen will, hatte zunächst ein vollständig negatives Resultat. Die Hauptbestandtheile der Phosphorwolframsäureniederschläge, die isolirt und, soweit nöthig, durch Analysen identificirt wurden, waren grosse Mengen Ammoniak, sehr viel Leucin, etwas Tyrosin und eine Amidosäure, die ein dem der Asparaginsäure ähnliches Cu-Salz gab, dessen Analysenwerthe aber, trotzdem das Salz durch mehrfaches Umkrystallisiren gereinigt war, weder für Asparaginsäure noch für eine andere bekannte Amidosäure stimmten, und das aus Mangel an Material nicht weiter untersucht werden konnte. ${ }^{1}$ )

Auf eine briefliche Anfrage antwortete mir Drechsel (am 13. 4. 1897), es sei ihm wohlbekannt, dass die Abscheidung der Basen bisweilen grössere Schwierigkeiten darbietet und dass die Ausbeute bei verschiedenen Eiweisskörpern verschieden zu sein scheint. Er spricht darauf die Vermuthung aus, dass vielleicht die verschiedene Zeitdauer des Kochens - bei Drechsel $3 \times 24$ Stunden, bei mir 5 Stunden - eventuell auch die verschiedene Stärke der verwendeten Salzsäure auf die Entstehung der Basen von Einfluss sei, und hält es für möglich, sogar für wahrscheinlich, dass die Basen nicht unmittelbar entstehen, sondern dass zunächst eine noch complicirtere Verbindung sich bildet, die erst später in Lysin etc. zerfällt. Im Uebrigen empfahl er mir zur Abscheidung des Lysins die Benzoylchloridmethode als sehr geeignet und gab mir für die Anwendung derselben eine genaue Vorschrift, die ich hier, da sie von ihm nicht mehr hat publicirt werden können, wörtlich folgen lasse. Sie enthält einige Abweichungen gegenüber der von Clara Will den ow kürzlich veröffentlichten, ${ }^{2}$ ) ist aber später niedergeschrieben, als die Angaben, auf welche sich Willdenow stützt, und daher jedenfalls als die besser von Drechsel in ihren Einzelheiten ausgearbeitete Methode anzusehen.

1) Entgegen der allgemeinen Annahme gehen ganz erhebliche Mengen von Amidosäuren in den Phosphorwolframsäureniederschlag über, ein Verhalten, das noch näherer Untersuchung bedarf.

2) Zeitschr. f. physiol. Chem., Bd. XXV, S. 523. 
"Die Benzoylirung nach Schotten-Baumann erfolgt leicht und glatt; das Produkt ist die Lysursäure (Dibenzoyllysin), die in der alkalischen Flüssigkeit gelöst bleibt. Man erhitzt diese auf dem kochenden Wasserbade und versetzt heiss mit $\mathrm{HCl}$ bis zur deutlichen Reaction auf Congo, worauf man auf dem Wasserbade erkalten lässt. Die Lysursäure fällt als Oel aus, die Benzoesäure krystallisirt beim Erkalten aus und kann abgeschlämmt werden. Zweckmässig reinigt man die rohe Lysursäure zunächst durch Behandeln mit heissem Wasser oder durch Umfällen, dann aber löst man sie in heissem überschüssigen Barytwasser, filtrirt, fällt mit $\mathrm{CO}_{2}$, kocht, filtrirt wieder und versetzt nun das klare, neutrale Filtrat kochend heiss und unter gutem Umschütteln mit kleinen Mengen verdünnter Salzsäure, bis eine Krystallisation beginnt. Jeder Tropfen Salzsäure erzeugt zunächst eine milchige Trübung, die beim Umschütteln verschwindet und nach einiger Zeit einer krystallinischen Fällung Platz macht; setzt man zuviel HCl zu (bis zur Reaction auf Congo), so bekommt man eine ölige Fällung, die man aber durch Barytwasser wieder lösen kann. Hat man den Punct richtig getroffen, so erstarrt die Lösung oft schon in der Hitze zu einem dicken Krystallbrei, da der saure lysursaure Baryt auch in kochendem Wasser nur sehr schwer löslich ist, in kaltem fast gar nicht. Das bei $110^{\circ}$ getrocknete, oder einfacher bei $145-150^{\circ}$ geschmolzene Salz hat die $\mathrm{Zu}$ sammensetzung: $2 \mathrm{C}_{6} \mathrm{H}_{12}\left(\mathrm{C}_{7} \mathrm{H}_{5} \mathrm{O}\right)_{2} \mathrm{~N}_{2} \mathrm{O}_{2}+\left(\mathrm{C}_{6} \mathrm{H}_{11}\left[\mathrm{C}_{7} \mathrm{H}_{5} \mathrm{O}\right]_{2} \mathrm{~N}_{2} \mathrm{O}_{2}\right)_{2} \mathrm{Ba}$ und enthält $8,85 \% \mathrm{Ba}$.

Ich habe nun unter möglichst genauer Einhaltung der Drechsel'schen Vorschrift die mir aus den 3 Phosphorwolframsäurefällungen verbliebenen Rückstände, aus denen das $\mathrm{NH}_{3}$, Tyrosin, Leucin und die oben erwähnte Amidosäure entfernt waren, vereinigt und auf Lysin verarbeitet. Die Lösung wurde mit $750 \mathrm{ccm}$. 10\% iger Natronlauge versetzt und allmählich unter starkem Umschütteln bis zum jedesmaligen Verschwinden des Geruchs 100 gr. Benzoylchlorid zugesetzt. Aus der alkalischen Lösung schied sich zunächst ein schmieriger Antheil ab, der abfiltrirt wurde. Er war in Alkohol leicht löslich, war aber nicht zum Krystallisiren $\mathrm{zu}$ bringen und wurde noch nicht 
weiter untersucht. Das Filtrat wurde auf dem Wasserbade erhitzt, heiss $\mathrm{HCl}$ bis zur Reaction auf Congo zugefügt und auf dem Wasserbade erkalten gelassen. Es scheidet sich ein brauner, harzartiger Bodensatz und darüber grössere Mengen Benzoesäure ab, welche abgeschlämmt wurden. Das Harz wird noch zweimal mit Wasser ausgekocht, aus dem nach dem Erkalten noch reichlich Benzoesäure ausfällt, und dann in kochendem Barytwasser gelöst, filtrirt, $\mathrm{CO}_{2}$ durchgeleitet, gekocht, das neutrale, klare Filtrat kochend heiss mit sehr verdünnter Salzsäure versetzt. Gegen die Erwartung schied sich jedoch nichts Krystallinisches aus, nach dem Abkühlen bedeckt den Boden nur etwas schmieriges Harz. Die klar abgegossene Flüssigkeit wird wiederum kochend mit verdünnter $\mathrm{HCl}$ versetzt, nach dem Abkühlen von dem wieder nur ausgeschiedenen hellbraunen Harz abgegossen und nochmals kochend mit verdünnter $\mathrm{HCl}$ versetzt. Auch jetzt war noch keine Reaction auf Congo aufgetreten, es genügte aber, wie eine Probe zeigte, ein kleiner Zusatz von $\mathrm{HCl}$, um dieselbe hervorzurufen. Krystalle waren kein Mal erhalten. Abscheidung 2 und 3 wurden nun gesondert nochmals in Barytwasser gelöst und nach der Vorschrift behandelt, $\mathrm{HCl}$ nur soweit zugefügt, dass noch keine Congoreaction entstand. Wieder wurden nur Harze erhalten. Als jedoch jetzt die Mutterlauge von 2 auf Harz 3 + seiner Mutterlauge gegossen und gekocht wurde, schieden sich plötzlich Krystalle aus, lange Nadeln, von denen nach dem Abkühlen das ganze Kölbchen erfüllt war. Sie wurden abfiltrirt, von einem kleineren, noch harzigen Antheil abgeschlämmt, mehrmals mit kaltem Wasser ausgewaschen, wogen lufttrocken 1,7 gr. Die noch übrigen harzigen Antheile wurden zusammen nochmals in Barytwasser gelöst, aber in stärkerer Verdünnung, und mit $\mathrm{HCl}$ behandelt. Sie lieferten ausser Harz noch 1 gr. der Krystalle. Dieselben sind in Alkohol löslich, scheiden sich auf Zusatz des gleichen Volumens Wasser allmählich in Nadeln aus.

Die zuerst erhaltenen 1,7 gr. des Barytsalzes wurden aus heissem Wasser, in dem sie sich sehr schwer lösen, umkrystallisirt, beim Abkühlen entsteht zunächst eine milchige Trübung, die später schön krystallinisch wird. Es sind mi- 
kroskopisch lange, feine Nadeln, deren Schmelzpunkt bei $153^{\circ}$ liegt.

\section{Barytbestimmung.}

$0,4573 \mathrm{gr}$. werden im Platintiegel mehrere Stunden bei $150^{\circ}$ getrocknet. Die Substanz schmilzt dabei, nach dem Abkühlen bleibt ein schwach gelblich gefärbter Lack, der $0,4366 \mathrm{gr}$. wiegt. Verloren $=0,0207 \mathrm{gr} . \mathrm{H}_{\mathbf{2}} \mathrm{O}=$ $4,5 \%$. Die Substanz wird hierauf mit concentrirter $\mathrm{H}_{2} \mathrm{SO}_{4}$ abgeraucht, geglüht, nochmals mit $\mathrm{HCl}$ - befeuchtet, mit verdünnter $\mathrm{H}_{2} \mathrm{SO}_{4}$ versetzt, getrocknet und stark geglüht. Man erhält 0,0645 gr. $\mathrm{BaSO}_{4}=0,03792 \mathrm{Ba}=$ $8,7 \%$. Saurer lysursaurer Baryt verl. 8,83\% Ba.

\section{N-Bestimmung nach Dumas.}

0,2194 gr. der exsiccatortrockenen Substanz (verlor bei $110^{\circ}$ nur noch $\left.0,6 \% \mathrm{H}_{8} \mathrm{O}\right)$ gab $14,4 \mathrm{ccm}$. $\mathrm{N}$ bei $17^{\circ}$ und $764 \mathrm{~mm}$. Bar.

$\mathrm{N}=0,01680192=7,6 \%$. Verl. $\mathrm{N}=7,3 \%$.

Wurde der Rest des Barytsalzes in kochendem Wasser gelöst und heiss mit verdünnter $\mathrm{H}_{2} \mathrm{SO}_{4}$ in kleinem Ueberschuss versetzt, dann schnell filtrirt, so scheidet sich aus dem klaren Filtrat beim Abkühlen eine milchige Trübung aus, die sich zu Boden setzt und nach mehrtägigem Stehen allmählich in schöne Krystalldrusen umwandelt, die aus länglichen Blättchen bestehen, anscheinend freier Lysursäure. Dieselben schmelzen bei $150^{\circ}$. Für eine genauere Untersuchung war nicht mehr genügend Material vorhanden.

Ob bei der von mir benutzten Methode der Caseinspaltung wirklich nur so geringe Mengen basischer Bestandtheile vorhanden sind, als ich sie erhielt, vermag ich nicht zu sagen, es bedarf dazu jedenfalls einer nochmaligen Untersuchung; allem Anscheine nach sind jedoch grössere Mengen Lysin vorhanden, z. B. wandelte sich eine der harzigen Ausscheidungen des sauren lysursauren Baryts noch nach 14 tägigem Stehen zum Theile in Krystalle um. Jedenfalls sind die Bedingungen, unter denen der saure lysursaure Baryt zum Krystallisiren zu bringen ist, nicht so leicht zu beherrschen, als es nach der Drechselschen Vorschrift den Anschein hat.

Die Verarbeitung des nach dem Abdampfen des Alkohols erhaltenen letzten Rückstandes auf die darin ausser den Basen 
enthaltenen Bestandtheile stiess zunächst auf grosse Schwierigkeiten. Der in einem Versuch aus 325 gr. Casein erhaltene Rückstand, der 65 gr. wog, wurde heiss in absolutem Alkohol gelöst, nach einigen Tagen schied sich nur etwas Leucin ab, von dem abfiltrirt wurde. Das Filtrat wurde abdestillirt, es schied sich nichts aus; beim Versetzen mit Wasser erhält man noch geringe Mengen des Pyridinderivats. Um das im Filtrat anscheinend in grossen Mengen enthaltene Leucin zu gewinnen, wurde das Ganze in $3 \mathrm{l}$. Wasser gelöst, gekocht und frisch gefälltes $\mathrm{Cu}(\mathrm{OH})_{2}$ eingetragen, bis sich nichts mehr löste, das dunkelblaue Filtrat auf kleines Volumen eingedampft. Es scheidet sich jedoch nicht die Spur Leucinkupfer aus. Das Cu wurde darauf durch $\mathrm{H}_{2} \mathrm{~S}$ entfernt, das schwach gelblich gefärbte Filtrat auf ca. $20 \mathrm{ccm}$. eingedampft. Selbst nach mehrmonatlichem Stehen wurde die Masse nicht fest, es trat in dem Syrup nur eine Ausscheidung von garbenförmigen Nadeln (wahrscheinlich Tyrosin) und blassen Kugeln (wohl Leucin) ein. Nachdem auch eine Reihe anderer Versuche, deren nähere Beschreibung zwecklos wäre, resultatlos verlaufen war, gelangte ich schliesslich auf folgende Weise zum Ziele:

Das bei der Verarbeitung auf Basen gewonnene Filtrat des 3. Phosphorwolframsäureniederschlages des aus $21 / 2 \mathrm{~kg}$. Casein erhaltenen letzten Rückstandes (siehe oben), ca. 26 l., wurde mit starkem Barytwasser von der überschüssigen Phosphorwolframsäure befreit und $\mathrm{zu}$ der schon stark alkalischen Flüssigkeit noch so viel $\mathrm{Ba}(\mathrm{OH})_{2}$ zugesetzt, dass beim Durchleiten von $\mathrm{CO}_{2}$ etwas überschüssiger Baryt ausfiel - es wurde dazu etwa $1 \mathrm{~kg}$. fester Aetzbaryt verbraucht -, darauf der überschüssige Baryt durch $\mathrm{CO}_{2}$ entfernt und das Filtrat, also das Barytsalz aller vorhandenen Stoffe, auf etwa 2 l. eingedampft und von etwas nachträglich ausgeschiedenem $\mathrm{BaCO}_{3}$ abfiltrirt. Beim weiteren Einengen zum dünnen Syrup schieden sich grosse Massen eines Barytsalzes ab (I), das aus mikroskopisch kleinen spitzen Nadeln besteht. Es wird abfiltrit und mit etwas kaltem Wasser ausgewaschen. Die Mutterlauge gibt bei weiterem Einengen eine zweite Abscheidung (II). Deren Mutterlauge ist schon sehr dickflüssig und wird nicht weiter 
eingeengt. (Barytsalz III.) Barytsalz I wird in viel heissem Wasser gelöst und der Baryt durch $\mathrm{H}_{2} \mathrm{SO}_{4}$ genau ausgefällt, das Filtrat mit $\mathrm{Ag}_{2} \mathrm{O}$ zur Entfernung der letzten Spuren $\mathrm{Cl}$ geschüttelt, das Filtrat mit $\mathrm{H}_{2} \mathrm{~S}$ entsilbert und das nun erhaltene Filtrat auf ca. $50 \mathrm{ccm}$. eingedampft. Es scheidet sich eine grosse Menge Krystalle aus, die fast nur aus langen Nadeln, zum Theile dicken prismatischen Krystallen bestehen. Sie werden abfiltrirt, reichlich mit kaltem Wasser ausgewaschen, in dem sich kaum etwas löst. Sie sind auch in heissem Wasser schwer löslich, geben sehr starke Millon'sche Reaction, schmelzen bei $293^{\circ}$. Es handelt sich um Tyrosin, dessen Menge 6 gr. betrug. Das Filtrat gab noch schwache Millon'sche Reaction. Es wird zusammen mit dem Waschwasser auf $20 \mathrm{ccm}$. eingedampft. Dabei scheiden sich zunächst 5,5 gr. Leucin ab, aus dessen Filtrat man bei weiterem Einengen noch 2,8 gr. und nochmals 7,7 gr. Leucin erhält. Das Filtrat der letzteren, welches starke Millon'sche Reaction gibt, während die 3 Leucinfractionen sie nur sehr schwach zeigen, wird weiter bis zum etwas dickflüssigen Syrup, auf etwa $10 \mathrm{ccm}$., eingeengt, nach mehrtägigem Stehen unterm Exsiccator scheiden sich noch 5 gr. Leucin ab. Deren Filtrat wird etwas mit Wasser verdünnt, mit Thierkohle entfärbt und an der Luft stehen gelassen. Es scheiden sich allmählich noch geringe Mengen Tyrosin $a b$, nach deren Abfiltriren eine syrupöse Mutterlauge bleibt, die unterm Exsiccator sich etwas eindickt, aber nichts Festes mehr absetzt. Es sind etwa 2 bis 3 ccm., die in Alkohol löslich sind und noch Millon'sche Reaction geben. Barytsalz I bestand also fast ganz aus Tyrosin (über $6 \mathrm{gr}$.) und Leucin (21 gr.).

Barytsalz II, das in geringerer Menge erhalten war, wird in derselben Weise behandelt, liefert aber trotz positiver Millon'scher Reaction keine Tyrosinabscheidung. Man erhält durch fractionirtes Eindampfen 1,5+1,5+5,7=8,7 gr. Leucin. Das letzte Filtrat wird unterm Exsiccator noch theilweise krystallinisch von ausgeschiedenem Leucin, das sich nicht mehr gut abfiltriren lässt. Es handelte sich im Ganzen bloss noch um wenige Cubikcentimeter.

Die Lösung von Barytsalz III hatte nach mehrmonatlichem Stehen nur minimale Mengen Krystalle von dem Ver- 
halten des Barytsalzes, II abgeseizt, von denen abfiltrirt wird. Da es mir aussichtslos erschien, aus dem schon dickflüssigen Syrup durch weiteres Einengen etwas Greifbares zu gewinnen, so fällte ich den Baryt durch $\mathrm{H}_{2} \mathrm{SO}_{4}$ genau aus, wozu etwa $300 \mathrm{ccm}$. 20\% iger $\mathrm{H}_{2} \mathrm{SO}_{4}$ erforderlich waren. Das $\mathrm{Ba}-$ und $\mathrm{H}_{2} \mathrm{SO}_{4}$-freie Filtrat wird nach Entfärben mit Thierkohle zu einem dünnen, hellgelben Syrup eingedampft, der nicht krystallisirt. Eine Probe wird bei weiterem Eindampfen auch nicht krystallinisch, ebensowenig ein aus ihr dargestelltes Zinksalz, das auf Zusatz von Alkohol und Aether ölig ausfällt. Die daraus zurückgewonnene freie Säure, auf dem Wasserbade eingedampft und über $\mathrm{H}_{2} \mathrm{SO}_{4}$ längere Zeit getrocknet, wird lackartig, löst sich leicht in Alkohol, wird daraus durch Aether ölig gefällt. Nach diesen und verschiedenen anderen fruchtlosen Versuchen, die Säure oder Salze derselben zum Krystallisiren $\mathrm{zu}$ bringen, kam ich endlich in folgender Weise zum Ziele: ich versetzte die Masse mit viel concentrirter $\mathrm{HCl}$, worin sie sich leicht löste, und dampfte sie zu einem Syrup ein. Derselbe erstarrte innerhalb 24 Stunden zu einem dicken Krystallbrei, der mit der Saugpumpe abgesogen, noch dreimal mit frischen Portionen concentrirter $\mathrm{HCl}$ verrieben und immer wieder filtrirt wurde, bis ein fast farbloses Krystallpulver zurück-. blieb. Dieses wird zur weiteren Reinigung in etwa $100 \mathrm{ccm}$. concentrirter $\mathrm{HCl}$ unter Sieden gelöst, durch Glaswolle klar filtrirt. Aus der schwach bräunlich gefärbten Flüssigkeit scheiden sich bei langsamem Abkühlen in etwa einer Stunde feste Krystalldrusen $a b$, die aus über $1 \mathrm{~cm}$. langen, dicken prismatischen Krystallen bestehen. Es sind nach dem Auswaschen mit Salzsäure und Trocknen an der Luft $43 \mathrm{gr}$. Aus ihrer Mutterlauge erhält man bei weiterem Einengen zum Syrup noch 10 gr. derselben Krystalle, deren Mutterlauge bei weiterem Eindampfen nur noch etwa $3 \mathrm{ccm}$. beträgt und nicht mehr krystallisirt. Aus verdünnter Salzsäure scheiden sich die Krystalle in grossen, glänzenden, durchsichtigen, dicken rechteckigen Tafeln ab. Ein kleiner Theil der Krystalle wird in Wasser gelöst, mit $\mathrm{Ag}_{2} \mathrm{O}$ von der Salzsäure befreit, das gelöste Silber durch $\mathrm{H}_{2} \mathrm{~S}$ entfernt und das Filtrat zur Trockne ver- 
dampft. Es bleibt ein krystallinischer Rückstand von reiner Glutaminsäure zurück, die bei $202^{\circ}$ schmilzt.

Sämmtliche Mutterlaugen der salzsauren Glutaminsäure werden wieder zum Syrup eingedampft. Derselbe ist schwarz gefärbt und erstarrt allmählich im Laufe mehrerer Wochen zu einem dicken Krystallbrei, der wiederum aus salzsaurer Glutaminsäure besteht. Aus ihrer Mutterlauge lassen sich durch weiteres Eindampfen noch 2 Portionen der salzsauren Glutaminsäure gewinnen. Die letzte Mutterlauge, die sehr dickflüssig ist, wird nach mehrmonatlichem Stehen nur zum geringen Theil krystallinisch und zwar scheiden sich nicht prismatische Nadeln, sondern nur Kugeln $a b$, die sich nicht gut trennen lassen. Sie wird nun in viel Wasser gelöst, die Salzsäure durch Kupferoxydulschlamm möglichst entfernt, das blaue Filtrat durch $\mathrm{H}_{2} \mathrm{~S}$ entkupfert, nach Verjagen des $\mathrm{H}_{2} \mathrm{~S}$ die letzten Spuren $\mathrm{HCl}$ durch $\mathrm{Ag}_{2} \mathrm{O}$ entfernt und das durch $\mathrm{H}_{2} \mathrm{~S}$-Behandlung entsilberte Filtrat auf etwa $100 \mathrm{ccm}$. eingedampft. Jetzt, nach Entfernung der grossen Massen Glutaminsäure, scheidet sich endlich viel reines Leucin $a b$, ebenso noch bei dreimaligem fractionirten Eindampfen. Die letzte Mutterlauge ist schon dickflüssig, von den ausgeschiedenen Krystallkugeln nicht gut zu trennen. Sie wird mit den letzten geringen Mutterlaugen, die aus Barytsalz I und II erhalten waren, vereinigt, in viel Wasser gelöst, mit Thierkohle entfärbt und wieder fractionirt eingedampft. Man erhält noch 2 Krystallabscheidungen, die sich nach einmaligem Umkrystallisiren als reines Leucin erweisen. Die vereinigten letzten dickflüssigen Mutterlaugen erstarren unter dem Exsiccator zu einem K̇rystallbrei von Kugeln, jedenfalls auch Leucin, die sich indessen nicht mehr gut absaugen lassen. Ihre Gesammtmenge beträgt nicht mehr wie 10 gr., die also aus dem letzten Rückstand von $2 \frac{1}{1 / 2} \mathrm{~kg}$. Casein als alleiniger, nicht krystallinischer Rest übrig blieben, wahrscheinlich aber bei weiteren Bemühungen auch noch zum Krystallisiren zu bringen wären. Alles Uebrige ist durch das von mir angewandte Verfahren in krystallinische Bestandtheile zerlegt worden und zwar bestand es aus geringen Mengen Tyrosin, grossen Massen Glutaminsäure und reichlichen Leucinmengen. 
Grössere Schwierigkeiten noch, als die Verarbeitung des letzten Rückstandes bereitete die Zerlegung der in Alkohol unlöslichen, früher von mir sogenannten öligen Säure. Nachdem ich mit ihrer Umwandlung in das Cu-Salz nicht zum Ziele gekommen war, löste ich die aus $2 \mathrm{~kg}$. Casein stammende Masse in kochendem Wasser, dabei blieb ein Theil ungelöst, der fast ganz aus Anorganischem bestand. Die Lösung (11/2 Liter) wurde nun fractionirt mit Bleiacetat gefällt, welches einen dicken, flockigen Niederschlag erzeugte. Ich erhielt 6 Fällungen durch je 15 gr. Bleiacetat in 10\% iger Lösung. Das letzte, nur wenig entfärbte Filtrat gab keine Fällung mehr. Die in Wasser suspendirten und durch $\mathrm{H}_{2} \mathrm{~S}$ zersetzten Niederschläge gaben beim Eindampfen geringe Mengen von Syrupen, die krystallinisch erstarrten und der Hauptsache nach aus anorganischen Salzen bestanden. Das Filtrat der letzten Bleifällung wurde durch $\mathrm{H}_{2} \mathrm{~S}$ entbleit, das Filtrat zur Verjagung der Essigsäure viermal unter Erneuerung des Wassers eingedampft, nochmals in Wasser gelöst, mit Thierkohle entfärbt und zu einem dünnen Syrup eingeengt, der auf keine Weise zum Krystallisiren zu bringen war, sodass ich die Einzelheiten dieser Versuche umgehen kann. Ich versuchte es nun mit der Ueberführung in ein salzsaures Salz. Der ganze Syrup wurde mit einem grossen Ueberschuss concentrirter $\mathrm{HCl}$ vermischt und $\mathrm{zu}$ einem mässig dicken Syrup eingedampft, der nach längerem Stehen nur etwas Anorganisches absetzte, das durch Glaswolle abfiltrirt wurde. Ein weiterer Zusatz von $\mathrm{HCl}$ und achttägiges Stehen bewirkten die Abscheidung eines mässigen Bodensatzes, der aus salzsaurer Glutaminsäure bestand. (S. die I. Mittheilung, S. 174, Anmerkung.) Eine Reihe weiterer Versuche, den Syrup zum Krystallisiren $\mathrm{zu}$ bringen, schlug wiederum fehl.

In der Annahme, dass derselbe vielleicht die Muttersubstanz einer krystallisirenden Säure enthalte, die beim blossen Kochen mit $\mathrm{HCl}$ noch nicht abgespalten wurde, löste ich ihn in $200 \mathrm{ccm}$. concentrirter $\mathrm{HCl}$, fügte $200 \mathrm{ccm}$. Wasser und 50 gr. Zinnchlorür zu und kochte das Gemenge 3 Tage lang. Die Lösung wurde in der üblichen Weise weiter verarbeitet, der schliesslich erhaltene salzsaure Syrup lieferte nach 3 Monate 
langem Stehen allmählich 3 gr. eines Bodensatzes, der aus salzsaurer Glutaminsäure bestand. Das Filtrat davon war nicht mehr zum Krystallisiren zu bringen.

Nachdem alle diese Versuche zur Trennung des Gemenges, um das es sich zu handeln schien, fehlgeschlagen, ging ich, in der Meinung, dass die Verarbeitung einer grösseren Menge vielleicht eher ein Resultat ergeben würde, in folgender Weise vor: Die aus $4 \mathrm{~kg}$. Casein einzeln erhaltenen 4 Antheile an der in Alkohol schwer Iöslichen Substanz, im Ganzen ca. 700 gr., wurden vereinigt in 2 l. Wasser unter Erwärmen gelöst, von einem ungelösten, hauptsächlich anorganischen Antheil abfiltrirt und noch warm mit der gleichen Menge concentrirter $\mathrm{HCl}$ vermischt. Nach Ablauf von 6 Wochen hatte sich nichts ausgeschieden. Die Lösung wird darauf auf $1^{1 / 2} \mathrm{l}$. eingedampft. Jetzt endlich beginnt nach einigen Tagen am Rande der Schale eine Krystallisation und allmählich wandelt sich der Inhalt in einen Krystallbrei um, der aus langen, rosettenförmig angeordneten Nadeln besteht. Sie wurden durch Glaswolle abzusaugen versucht, dies gelang indes nicht ganz vollständig, da das Filter sich allmählich verstopfte. Das erhaltene Filtrat lieferte bei weiterem Einengen nochmals reichlich Krystalle, die auch, soweit möglich, abfiltrirt wurden. Die auf den Filtern zurückgebliebene Masse, Gemenge der Krystalle mit ziemlich viel Mutterlauge, wird durch Erwärmen gelöst, filtrirt, erstarrt nach dem Abkühlen wieder zu einem dicken Krystallbrei, der sich durch Filtriren wiederum nicht abtrennen liess. Er wird jetzt in Wasser gelöst, filtrirt und zu einem dicken Syrup eingedampft. Nach mehrtägigem Stehen erstarrt derselbe zu einem weichen Krystallbrei, der auf Filtrirpapierplatten von der Mutterlauge möglichst befreit wird, die dem Papier dann durch Auslaugen mit Wasser wieder vollständig entzogen wird. Die Krystalle werden aus concentrirter $\mathrm{HCl}$ umkrystallisirt, man erhält so etwas über 200 gr. des salzsauren Salzes in fast farblosen Krystallen. Von einem kleinen Theil wird die freie Säure durch Behandlung mit $\mathrm{Ag}_{2} \mathrm{O}$ dargestellt, es resultiren Krystalle von reiner Glutaminsäure, deren Schmelzpunkt bei $203-204^{0}$ lag. 
Sämmtliche Mutterlaugen und die aus dem Filtrirpapier ausgelaugte Masse wurden vereinigt, mehrmals mit Wasser abgedampft, mit Thierkohle möglichst entfärbt und das Filtrat auf Syrupdicke eingedampft, wobei es sich wieder sehr dunkel färbte. Nach mehrmonatlichem Stehen scheidet sich am Boden der Schale ein weicher, wieder aus salzsaurer Glutaminsäure bestehender Krystallbrei aus, der indes durch Filtriren nicht rein zu erhalten war, da sich das Filter, nachdem ein Theil filtrirt war, verstopfte. Von dem ganzen Gemenge war etwa die Hälfte als klares Filtrat $\mathrm{zu}$ erhalten, die andere Hälfte, die den Krystallbrei enthielt, wurde nicht weiter verarbeitet. Das Filtrat wurde in viel Wasser gelöst, durch Kupferoxydul von der Salzsäure befreit, das Filtrat durch $\mathrm{H}_{2} \mathrm{~S}$ entkupfert und das nun fast tarblose Filtrat zu einem dicklichen Syrup (auf etwa $500 \mathrm{ccm}$.) eingedampft. Derselbe erstarrt nach mehreren Tagen allmählich zu einem Brei von Krystallen, die in ihrem Aussehen Leucinkugeln gleichen; sie liessen sich durch Filtriren nicht trennen. (Wurde eine kleine Probe des Gemisches in concentrirter $\mathrm{HCl}$ gelöst, dann die 3-5fache Menge Alkohol zugesetzt, wobei die Lösung klar blieb, und jetzt stark mit $\mathrm{NaOH}$ übersättigt, so schieden sich im Verlauf von 24 Stunden reichlich Krystalle aus, lange Nadeln, die ganz unorganisch sind und in denen $\mathrm{Ca}, \mathrm{CO}_{2}, \mathrm{H}_{2} \mathrm{SO}_{4}$, viel $\mathrm{H}_{3} \mathrm{PO}_{4}$ und Spuren $\mathrm{Cl}$ enthalten sind; die Hauptmasse ist wohl phosphorsaurer Kalk. Der Krystallbrei enthält also sehr viel Anorganisches, was wahrscheinlich mit der Grund für die schlechte Abscheidbarkeit der Krystalle ist.)

Der Krystallbrei wurde jetzt mit Alkohol übergossen, unter Alkohol öfters durchgeknetet, der Alkohol nach einigen Tagen abgegossen, erneuert und diese Procedur dreimal wiederholt; nach etwa 8 Tagen ist die Masse zum grössten Theil fest geworden. Nach der Trennung von der letzten alkoholischen Mutterlauge, die mit den andern beiden vereinigt wird, wird der Rückstand auf dem Wasserbade bis zur Gewichtsconstanz getrocknet, wobei die feste Masse wieder etwas zerfliesst. Sie wiegt 240 gr. Da sie nur aus etwa der Hälfte der Mutterlauge der salzsauren Glutaminsäure gewonnen ist, würden also in 
den 700 gr. der öligen Säure, die zur Verarbeitung kamen, ca. 480 gr. dieser Substanz enthalten gewesen sein, sodass, wenn ich die 200 gr. der salzsauren Glutaminsäure dazu rechne, auch die ölige Säure fast vollständig in krystallisirbare Produkte sich hat zerlegen lassen. Eine gänzliche Aufarbeitung der $240 \mathrm{gr}$. und der alkoholischen Mutterlaugen hat bisher noch nicht stattfinden können, ich habe erstere zwar schon in eine Reihe Fractionen zerlegt, dieselben aber, die neben Leucin als Hauptbestandtheil noch grosse Mengen anorganischer Substanzen enthielten, noch nicht definitiv erledigen können, was ich mir für später vorbehalte.

Ziehe ich in aller Kürze das Facit aus den mitgetheilten Versuchen, so glaube ich nachgewiesen zu haben, dass es sehr wohl möglich ist, die Spaltungsprodukte des Eiweisses quantitativ $\mathrm{zu}$ isoliren und bis auf einen minimalen Rest krystallisirt $\mathrm{zu}$ erhalten und $\mathrm{zu}$ identificiren. Wenn ich heute noch nicht in der Lage bin, ganz genau das gegenseitige quantitative Verhältniss der einzelnen Spaltungsprodukte anzugeben, so liegt das an der Schwierigkeit, in diesen ersten Versuchen, in denen zur Feststellung der geeigneten Methodik noch viel herumprobirt werden musste, ganz ohne Verluste zu arbeiten, was, wie ich hoffe, in den nächsten Versuchen gelingen wird. Ich kann daher z. B. die aus dem Casein abspaltbare Leucinmenge vorläufig nur schätzungsweise zu 40-50\% angeben und will auch heute über die Menge der Glutaminsäure, die von früheren Forschern schon auf etwa $30 \%$ berechnet ist und womit anscheinend das Richtige getroffen ist, mich einer bestimmten Meinungsäusserung enthalten. Jedenfalls aber glaube ich berechtigt zu sein, den von Kossel auf dem letzten Physiologencongress in Cambridge ${ }^{1}$ ) gethanen Ausspruch: "Die früher allein bekannten Spaltungsprodukte der Eiweisskörper, die Monoamidosäuren, haben sich einer quantitativen Bestimmung recht wenig zugänglich erwiesen» jetzt für nicht mehr zutreffend zu erachten.

Zum Schluss möchte ich noch über einen Spaltungsversuch berichten, den ich mit Hornsubstanz angestellt habe.

1) Deutsche med. Wochenschr. 1898, S. 581 . 
950 gr. feine, vom Drechsler bezogene Hornspäne wurden mit 4 1. concentrirter $\mathrm{HCl} 5$ Stunden gekocht. Die entweichenden Gase rochen nach $\mathrm{H}_{2} \mathrm{~S}$, aber auch mercaptanähnlich. In dem vorgelegten Wasser war wieder der Jodoform gebende Körper nachweisbar. Eine quantitative Bestimmung desselben mittelst Jods und unterschwefligsauren Natriums ergab auf Aceton berechnet ca. 0,1 gr., indessen ist jedenfalls mehr davon abgespalten, da das Wasser in der Vorlage sich durch die HClAbsorption sehr stark erwärmt und dabei wohl einen Theil der flüchtigen Substanz wieder entweichen lässt. Im Uebrigen wird die Verarbeitung in der gewöhnlichen Weise vorgenommen. Der Versuch ergab 43,5 gr. Tyrosin, also nicht erheblich mehr, als das Casein, auch sonst fanden sich ähnliche Verhältnisse. Ich muss jedoch erwähnen, dass dem ausgeschiedenen Tyrosin reichlich Krystalle in Briefcouvertform beigemengt waren, die, wie die Untersuchung ergab, aus oxalsaurem Kalk bestanden.

Das gesammte Tyrosin + den Krystallen wurde mit etwas ganz verdünnter $\mathrm{HCl}$ verrieben stehen gelassen, dann filtrirt und etwas ausgewaschen, das Filtrat mit essigsaurem Natrium versetzt. Neben Tyrosin fanden sich wieder viel Briefcouvertkrystalle. Auf Zusatz von $\mathrm{NH}_{3}$ löste sich das Tyrosin, die Briefcouvertkrystalle blieben zurück. Sie wogen 0,1885 gr. Eine Kalkbestimmung derselben ergab 20,6\% Ca, während oxalsaurer Kalk 31,3 Ca verlangt. Sie bestehen also nur zu zwei Dritteln aus oxalsaurem Kalk, das Uebrige waren noch Verunreinigungen. $1 \mathrm{~kg}$. Horn liefert demnach etwa $0,12 \mathrm{gr}$. oxalsauren Kalk. Nachträglich, als ich jetzt mehr darauf achtete, stellte sich heraus, dass auch bei der Caseinspaltung dem Tyrosin oxalsaurer Kalk beigemengt war. Die Angabe von D re chsel, dass bei der Eiweissspaltung durch Säuren, im Gegensatz zu der durch Alkalien, weder $\mathrm{CO}_{2}$ noch oxalsaurer Kalk abgespalten wird, beruht also auf einem Irrthum, und daher sind auch die von ihm daran geknüpften theoretischen Betrachtungen als nicht mehr zu Recht bestehend anzusehen. 